\title{
El mundo de los pobres en Gaudium et spes
}

\author{
RAMÓN SALA, OSA ${ }^{*}$
}

RESUMEN: La Constitución pastoral Gaudium et Spes es un documento peculiar e inacabado. Nace con la vocación de ser continuamente contextualizado y actualizado (GS 91b). Contempla a la Iglesia en el mundo de hoy. Uno de los escenarios de ese mundo de hoy es el de la pobreza. El presente artículo estudia la GS desde la perspectiva de los pobres. El compromiso con los pobres es una tarea permanente de la misión de la Iglesia y, hoy representa, además, un signo inequívoco de su credibilidad.

PALABRAS CLAVE: Vaticano II - Gaudium et Spes - pobres - mundo - recepción del Concilio.

ABSTRACT: The Pastoral Constitution Gaudium et Spes is a singular and unended document. It was born with the vocation of being continually contextualized and updated (GS 91b). It contemplates the Church in the World of Today. One of its stages is the poverty. This article studies GS under the poor's point of view. The commitment to the poor is a permanent task in the mission of the Church and, nowadays, it represents as well an unequivocal sign of her credibility.

KEY WORDS: Vatican II - Gaudium et Spes - poor - world - reception of the Council.

La Constitución pastoral Gaudium et Spes (GS) es el documento más extenso del Concilio Vaticano II y uno de los más complejos producidos por el magisterio conciliar de la Iglesia. Una Constitución singular tanto

\footnotetext{
* Profesor del Estudio Teológico Agustiniano (Valladolid).
} 
en la forma como en el fondo. En la forma, porque no existe ninguna otra "Constitución Pastoral". En el fondo, porque aborda una temática nueva y variada. Se comprende que durante el Concilio, algunos Padres la denominaran, más o menos irónicamente, como el "famoso esquema XIII", el "esquema sin nombre" e incluso el "arca de Noé". Lo cierto es que medio siglo después de su promulgación, sigue suscitando un vivo interés. Los estudiosos del Vaticano II la consideran "clave interpretativa" de un Concilio que, por voluntad expresa de Juan XXIII, se autodefinió como "pastoral". La recepción del Vaticano II en España se ha producido primariamente desde la doctrina de la Constitución pastorall.

Comentando este documento conciliar, Luis González-Carvajal dice que sobre la GS "quedan algunas cosas por decir y muchas más por hacer"2. Cuenta al final de su libro que una de las primeras comunidades de S. Francisco de Asís poseía un manuscrito del NT, algo totalmente insólito en una época en que "un NT valía más que un caballo". Cierto día una pobre viuda fue a pedir limosna a los mendicantes. Como no tenían otra cosa que aquel ejemplar, sin dudarlo, Francisco le entregó el preciado tesoro de la comunidad para que lo vendiera, "porque mejor es hacer lo que dice la Biblia que leerla". Y concluye el autor: "Sin duda, también es mejor hacer lo que dice la GS que leerla (aunque, evidentemente, no hay que contraponer lo uno a lo otro)".

En la presente exposición me voy a limitar a hacer una lectura de lo que dice la cuarta Constitución del Vaticano II sobre un tema que no trata sistemáticamente, pero que la atraviesa de principio a fin, y que ha tenido una incidencia notable en estos 50 años de Postconcilio. Me refiero al tema de la Iglesia y la pobreza en el mundo. Pero antes, como premisa, es preciso recordar muy brevemente la peculiaridad del documento que comentamos.

\section{Introducción: Peculiaridad de un documento inacabado}

GS resulta un documento singular desde distintos puntos de vista:

\section{A) Destinatarios}

Es el primer texto de un concilio dirigido expresamente no sólo a los fieles cristianos, sino "a todos los hombres" (GS 2a).

${ }^{1}$ Cf. R. BlázqueZ, "Postconcilio y eclesiología en España" en J.M. LABOA, ed., $E l$ postconcilio en España, Madrid 1988, 187-217; A. M. UNZUETA, El Vaticano II en una Iglesia local (Cuadernos Deusto 3), Bilbao 1995; H. RAGUER, Réquiem por la cristiandad. El Concilio Vaticano II y su impacto en España, Barcelona 2006.

2 L. González-CARVAJAl, Iglesia en el corazón del mundo, Madrid 2005, 17. 


\section{B) Metodología}

Es también el primer documento conciliar que utiliza un método inductivo ${ }^{3}$. Parte del análisis de la situación actual ("La condición del hombre en el mundo de hoy"), para desarrollar la exposición en dos partes: la primera de carácter doctrinal ("La Iglesia y la vocación del hombre") y la segunda destinada a atender "Algunos problemas más urgentes" (el matrimonio y familia, la cultura, la economía, la política y la paz).

\section{C) Proceso de elaboración}

Se trata del único documento del Vaticano II querido expresamente por el Papa Juan ${ }^{4}$, cuya temática no estaba prevista entre los esquemas de las comisiones preparatorias, y que se va a ver enriquecido con las orientaciones de todos los demás documentos fruto del Concilio: desde la concepción de la Iglesia (Lumen Gentium) hasta la del carácter inalienable de la dignidad humana (Dignitatis Humanae).

\section{D) Recepción}

Quizás la mayor novedad de este documento es la de llevar incorporada su recepción. Por primera vez en la historia de los concilios, GS fue promulgada con la conciencia de su no definitividad, es decir, de la necesidad de ser contextualizada y actualizada. Esta condición se explicita en el número que introduce la Conclusión de la Constitución pastoral:

Ciertamente, ante la inmensa diversidad de situaciones y de formas de cultura humana en el mundo, esta propuesta, en la mayoría de sus partes, presenta deliberadamente un carácter general; más aún, aunque pro-

${ }^{3}$ Lo había estrenado Juan XXIII en Mater et Magistra (1961). Consta de "tres fases: primera, examen completo del verdadero estado de la situación; segunda, valoración exacta de esa situación a la luz de los principios; y tercera, determinación de lo posible o de lo obligatorio para aplicar los principios de acuerdo con las circunstancias de tiempo y lugar. Son tres fases de un mismo proceso que suelen expresarse con estos tres verbos: ver, juzgar y actuar" (MM 236). Después de la Escritura, esta encíclica es la fuente más citada en GS (17 veces).

4 Dejó constancia de ello un mes antes de la solemne apertura del XXI Concilio Ecuménico en su célebre radiomensaje "Ecclesia Christi lumen gentium" (11 de septiembre de 1962). La idea de un documento específico sobre "Ia Iglesia ad extra" fue acogida por el Concilio al final del Primer Período de sesiones gracias a la importante intervención del card. Suenens (Malinas) en el aula conciliar (4 de diciembre). Al día siguiente recibió el apoyo decisivo del card. Montini, que cuando el Concilio reemprenda sus trabajos en su segunda etapa sería ya Pablo VI. 
clama la doctrina ya recibida en la Iglesia, dado que se trata no pocas veces de realidades sometidas a incesante evolución, deberá ser todavía continuada y ampliada (GS 91b).

Quiere decir que GS ha sido dada a las generaciones postconciliares para que sea recibida e interpretada, atendiendo, en primer lugar, "a la inmensa diversidad de situaciones y de formas de cultura humana en el mundo". En el espíritu de este párrafo, está ya presente la exigencia de inculturación, o mejor, de contextualización de la enseñanza del Concilio. En otras palabras, el proyecto de Iglesia ad extra trazado por el Vaticano II en GS necesita ser encarnado en las distintas latitudes de la Iglesia universal y atendiendo a las condiciones específicas de las Iglesias locales.

Y por otra parte, en segundo lugar, dado que se trata de "realidades sometidas a incesante evolución", la Constitución pastoral está llamada al aggiornamento, a ser continuamente puesta al día, releída, reinterpretada en atención a los nuevos signos de los tiempos. Obviamente el mundo de hoy no es el de los años 60'; los tiempos han cambiado.

Teniendo esto en cuenta, con la perspectiva de los 50 años transcurridos, nos interrogamos sobre los pobres en el mundo y en el hoy de la GS. ¿Qué tiene todavía que decir al respecto este documento conciliar?

No han faltado quienes han observado que GS contempló el mundo con una mirada "eurocéntrica" y en un momento histórico determinado para cuestionar su vigencia en otras circunstancias espacio-temporales. Se trataría de un documento occidental y obsoleto. Tan precipitada conclusión no resiste, sin embargo, un análisis riguroso del significado que la propia Constitución pastoral da a las nociones de "mundo" y de "hoy". En ella "mundo" es el mundo de los hombres, el género humano, la familia humana universal (cf. GS 2b). Se pone el acento no sobre el cosmos, sino sobre la humanidad. Tiene, por tanto, un valor antropológico y social universal más allá de las diferencias geográficas o culturales. Por otra parte, el "hoy" de GS tampoco es cronológico, sino cristológico: Cristo, el mismo ayer, hoy y siempre, alfa y omega, principio y fin de la historia humana (cf. GS $10 \mathrm{~b} ; 45)$. Cristo es el sentido de la totalidad del tiempo, en quien se recapitula el misterio de la historia. Esta es, en definitiva, la clave de su permanente actualidad" 51 mundo como "Teatro de la historia del géne-

5 Cf. A. NIRAPPEL, "Towards a Definition of the Term 'World' in Gaudium et Spes": Ephemerides Theologicae Lovanienses 48 (1972) 89-126; G. CAVIGLIA, "Gesù Cristo, 'punto focale dei desideri della storia e della civiltà' (GS 45), incontro affascinante tra eternità e tempo": Salesianum 54 (1992) 41-78. 
ro humano" es contemplado por la Constitución Pastoral en dos escenarios: el de la modernidad secularizada (el mundo del progreso, de la ciencia y la técnica) y el de los pobres (el mundo de las injusticias, de la exclusión y las desigualdades) ${ }^{6}$. A éste último escenario nos vamos a referir en las páginas que siguen?.

La exposición está dividida en dos partes. En la primera parte, siguiendo un procedimiento sincrónico, se retoman y comentan los principales textos de GS sobre la materia que nos ocupa. En la segunda, de carácter diacrónico, se recorren las etapas de su recepción en el Postconcilio. Para terminar se ofrecen algunas reflexiones conclusivas.

\section{1. "Los gozos y las esperanzas... sobre todo de los pobres"}

\subsection{Praesertim pauperum (GS 1)}

Los gozos y las esperanzas 8 , las tristezas y las angustias de los hombres de nuestro tiempo, sobre todo de los pobres y de todos los afligidos, son también gozo y esperanza, tristeza y angustia de los discípulos de Cristo y no hay nada verdaderamente humano que no tenga resonancia en su corazón (GS 1).

Ya las palabras iniciales del Proemio de la Constitución pastoral ponen de relieve que su mensaje universal se dirige en primer lugar a dar respuesta a las aspiraciones y al dolor "sobre todo de los pobres y de todos los afligidos". Son ellos los que mejor encarnan para la Iglesia las esperanzas y las angustias de la humanidad en el mundo actual ${ }^{9}$. A lo largo de la primera parte del documento esta atención prioritaria a los pobres (se 388

6 Cf. S. MADRIGAL, Unas lecciones sobre el Vaticano II y su legado, Madrid 2012, 383-

7 Cf. R. SAla, "En el mundo por los pobres. El legado de Gaudium et spes" en I. GonZÁlez Marcos, Concilio Vaticano II. 40 años después. IX Jornadas Agustinianas, Madrid 2006, 153-189.

8 Aquí hemos preferido usar el plural de la expresión original francesa (Les joies et les espoirs), aunque en el texto oficial latino se use el singular. Pero, salvo que se indique otra cosa, se citan los textos de GS según la edición bilingüe de la Conferencia Episcopal Española (Concilio Ecuménico Vaticano II. Constituciones. Decretos. Declaraciones, Madrid 1993, 298-499).

9 La perspectiva de la esperanza de la humanidad preside todo el documento según la intención manifestada por los redactores de la subcomisión central que en Ariccia (esq. III) decidieron modificar significativamente el orden de la formulación inicial del texto de Zurich (esq.II): se sustituye "Gaudium et luctus, spes et angor". (Acta Synodalia III, V, 116) por "Gaudium et spes, luctus et angor" (Ibid., IV, I, 435). 
usa el sinónimo egentes) como destinatarios de la misión de la Iglesia en el mundo reaparece de nuevo de forma expresa en otras dos ocasiones: GS 21e y 42b. En este último número, perteneciente al cap. 4, que Congar llama "el corazón" de la GS10, a propósito de "la ayuda que la Iglesia procura ofrecer a la sociedad humana", el texto señala que la Iglesia no se identifica con ningún sistema político, económico o social porque su "misión propia" es de orden religioso -obsérvese que no dice "espiritual", sino "religioso"-. Ahora bien, precisamente en razón de ese fin, "según las circunstancias de tiempo y lugar", esa misión incluye el deber de promover acciones de carácter temporal sobre todo al servicio de los pobres (GS 42b). También en el orden político cuando se trata de defender la dignidad de la persona humana y sus derechos fundamentales, como especificará más adelante la propia Constitución pastoral ${ }^{11}$. El principio enunciado en este pasaje constituye el punto de partida de un itinerario que conducirá, inmediatamente después del Concilio, a la opción preferencial de la Iglesia por los pobres.

Asumiendo la perspectiva hermenéutica desarrollada por la propia Constitución pastoral con su método inductivo, móstramos a continuación: el análisis descriptivo de la realidad de los pobres, el fundamento cristológico que ilumina esa realidad y las pautas de acción que propone la Iglesia, tal y como se exponen en GS. Como se podrá comprobar el lema "especialmente... los pobres", de modo explícito (cf. GS 27b, 57f, 69, 84a, $86 \mathrm{c}$ ), sirve de hilo conductor.

\subsection{Un signo de los tiempos}

La Constitución pastoral esboza en GS 4 una descripción general de los rasgos que caracterizan la situación del mundo. Junto a los "profundos y rápidos cambios" (GS 4b) y a la desorientación que provoca el progre-

10 Adopta el mismo título general del documento anteponiéndole la expresión " $D e$ munere" y es efectivamente el puente entre la primera y la segunda parte. Cf. Y. CoNGAR, "El papel de la Iglesia en el mundo de hoy ( $1^{a}$ parte, capítulo IV)" en Y. CoNGAR - M. PEUCHMAURD, eds., Vaticano II. La Iglesia en el mundo de hoy. Constitución pastoral 'Gaudium et spes' II, Madrid 1970, 373-403.

11 GS 76e: "Ciertamente, las realidades temporales y las que en la condición humana transcienden este mundo están estrechamente unidas entre sí, y la Iglesia se sirve de medios temporales cuando su propia misión lo exige. No pone, sin embargo, su esperanza en privilegios otorgados por la autoridad civil... Pero, la Iglesia debe poder, siempre y en todo lugar... emitir un juicio moral también sobre cosas que afectan al orden político cuando lo exijan los derechos fundamentales de la persona o la salvación de las almas...". 
so en los campos del poder y saber humanos y en las normas que rigen la sociedad (GS 4c), describe como uno de "signos de los tiempos"12 el clamor de los pobres (GS 4d). Lo hace por medio de contrastes: entre las enormes posibilidades de bienestar para todos y las mayorías que continúan sin cubrir las necesidades básicas ("todavía una enorme parte de la población mundial se ve afligida por el hambre y la miseria y es incalculable el número de los totalmente analfabetos"); entre la conciencia universal de la propia libertad y la aparición de "nuevos tipos de esclavitud social y psicológica"; entre la percepción del mundo como una "aldea global" ("la mutua interdependencia de unos con otros dentro de la necesaria solidaridad") y los conflictos (políticos, sociales, económicos, raciales e ideológicos) que dividen y amenazan a la humanidad.

Después de constatar la brecha existente "entre las categorías sociales de diverso género" y "entre naciones ricas, menos desarrolladas y pobres", la misma Exposición introductoria ofrece en GS 9 una magnífica descripción que ilustra de una forma gráfica quienes son los pobres a los que se refiere la Constitución pastoral:

De ahí que muchos exijan insistentemente aquellos bienes de los que se consideran privados por la injusticia o por un reparto no equitativo. Los países en vías de desarrollo... quieren participar de los beneficios de la civilización moderna, no sólo en el campo político, sino también en el económico, y desempeñar libremente su papel en el mundo. Sin embargo, aumenta cada día su distancia y muchas veces su dependencia, incluso económica, con respecto a las naciones más ricas que avanzan más rápidamente. Los pueblos que pasan hambre interpelan a los más opulentos. Las mujeres reivindican, allí donde aún no lo han conseguido, la igualdad de derecho y de hecho con los hombres. Los obreros y campesinos desean no sólo adquirir lo necesario para alimentarse, sino desarrollar mediante su trabajo sus dotes personales y participar en la ordenación de la vida económica, social, política y cultural. Ahora, por primera vez en la historia humana, los pueblos están persuadidos de que los beneficios de la cultura pueden y deben extenderse realmente a todos (GS 9b).

12 En el documento hay otras alusiones más o menos explícitas (cf. GS 11a; 44b; 50b; $76 \mathrm{ce} ; 87 \mathrm{c}$; etc.). Se debe a Pacem in terris (1963) la introducción de la categoría evangélica de los "signa temporum" en el lenguaje del Magisterio. En esa encíclica designan en particular tres "fenómenos que caracterizan la época moderna": la promoción económica y social de las clases trabajadoras, el ingreso de la mujer en la vida pública y la nueva configuración política mundial (PT 21-25). Tanto GS como los demás documentos del Concilio (cf. PO 9b, AA 14c, UR 4a) emplean la expresión en el sentido moderno indicado. 
Lejos de dar definiciones teóricas, este párrafo identifica a los “pobres" bajo una doble caracterización. En un primer nivel, englobando a poblaciones enteras del planeta: "los pueblos que pasan hambre". Después se refiere igualmente a determinados colectivos marginados, en particular, las mujeres, el mundo obrero y los trabajadores del campo. Por otra parte, su condición se presenta también distinguiendo dos indicadores: los bienes económicos y los bienes culturales, "de los que se consideran privados por la injusticia o por un reparto no equitativo". Desde el punto de vista económico, se subraya que aumenta la dependencia de los países pobres con respecto a los más ricos, y por otro lado, la legítima aspiración de las clases trabajadoras a participar en la ordenación de la vida económica. Desde el punto de vista del acceso a la cultura, se pone de relieve la convicción de que los beneficios de la civilización moderna deben ponerse al alcance de todos sin exclusiones.

\subsection{Cristo mismo clama en los pobres (GS 88)}

Varios pasajes de la Constitución pastoral iluminan a la luz de la Palabra de Dios la misión de una Iglesia comprometida en favor de los pobres. Recurriendo a textos neotestamentarios de claro contenido social, GS 27a, inculcando "el respeto a la persona humana", insiste en el cuidado y la cercanía de la Iglesia a los más necesitados como una exigencia práctica y urgente. Primero, con la alusión implícita (nota 8) a dos versículos de la Epístola de Santiago (cf. Sant 2,15-16) y la referencia a la "parábola del pobre Lázaro" (nota 9, cf. Lc 16,19-31). Y a continuación, en el párrafo siguiente, con la cita textual del mensaje central de la "parábola del juicio final" (cf. Mt 25,31-46). Estamos ante la perícopa clave, según GS, para entender la predilección de la Iglesia por los pobres.

En nuestros días sobre todo, urge la obligación de acercarnos a cualquier otro hombre y servirle activamente cuando llegue la ocasión, ya se trate de un anciano abandonado por todos, de un trabajador extranjero injustamente despreciado, de un desterrado o de un niño nacido de una unión ilegítima..., del hambriento que interpela nuestra conciencia, recordándonos la palabra del Señor: Cuantas veces hicisteis esto a uno de mis hermanos más pequeños, a mí me lo hicisteis (Mt 25,40) (GS 27b).

Hay que observar que este pasaje comienza utilizando de nuevo el adverbio praesertim con la connotación teológica que tiene para nuestro tema, como ya se ha indicado. Sigue una enumeración de algunos "rostros de la pobreza" (ancianos, inmigrantes, exiliados, niños, hambrientos), sin otra pretensión que la de traducir con ejemplos actuales, el perfil de los 
sujetos concretos con los que Cristo se identifica personalmente hoy al hilo de la parábola evangélica.

"La Iglesia... descubre en los pobres y en los que sufren la imagen de su fundador, pobre y sufriente, se preocupa de aliviar su miseria y busca servir a Cristo en ellos..." (LG 8c). También la segunda parte de GS reconoce claramente el fundamento cristológico de la tarea de la Iglesia en el mundo de los pobres (cf. GS 72ab). La identificación de Cristo con el clamor de los pobres constituye el tema central de GS 88. Es un texto que se encuentra en el último capítulo de la segunda parte (cap. 5: El fomento de la paz y la promoción de la comunidad de los pueblos). Lleva el poco ingenioso título "Tarea de los cristianos en la prestación de ayudas". Pero ahí se afirma de modo elocuente que:

Los cristianos deben cooperar de buen grado y de todo corazón en la construcción de un orden internacional con el respeto auténtico de las libertades legítimas y la amistosa fraternidad de todos, tanto más cuanto que la mayor parte del mundo sufre todavía tan gran necesidad, que el mismo Cristo parece elevar su voz en los pobres para provocar la caridad de sus discípulos. Por consiguiente, que no sirva de escándalo para los hombres que algunas naciones, cuya población muchas veces es en su mayoría cristiana, tengan abundancia de bienes, mientras otras se ven privadas de lo necesario para vivir, atormentadas por el hambre, las enfermedades y todo tipo de miseria. Pues el espíritu de pobreza y el de caridad son gloria y testimonio de la Iglesia de Cristo (GS 88a).

En el llamado "sacramento del pobre" se significa el modelo por excelencia de la responsabilidad hacia el hermano necesitado. De aquí GS extrae auténticos imperativos que apelan al compromiso social de los cristianos.

\subsection{El compromiso por los pobres}

Aplicando "los principios y las luces que manan de Cristo" (GS 46b) a la problemática concreta del mundo actual en sus diversos ámbitos (familiar, cultural, socio-económico, político e internacional), toda la segunda parte de la Constitución pastoral se presenta como un "programa de praxis"13.

Naturalmente el capítulo 3 ("La vida económico-social") ofrece abundantes orientaciones en este sentido, retomando las directrices del magisterio pontificio en materia económica (cf. GS 63-72). Pero el com-

13 Y. CONGAR, "El papel de la Iglesia en el mundo de hoy", 375. 
promiso de la Iglesia con los pobres es subrayado con especial énfasis a lo largo del capítulo 5, al tratar de la paz ${ }^{14}$.

Analizando las causas de las guerras, se recuerda que para construir la paz hay que erradicar las injusticias y que "no pocas de ellas provienen de las excesivas desigualdades económicas" (GS 83). La pobreza no es sólo la consecuencia de las guerras, sino también una de sus causas principales. Hoy diríamos que no hay tráfico de armas en los países pobres porque haya guerras; sino al revés, que hay guerras en los países pobres porque se gasta en armamento lo que no se gasta en otras cosas. Se reclama también la necesidad de un ordenamiento internacional que proteja el bien común universal, sobre todo (prasertim) en "numerosas regiones que sufren todavía una miseria intolerable". A tal fin, se insta a los organismos internacionales a adoptar medidas globales tendentes a cubrir las necesidades básicas (alimenticias, sanitarias, educativas, laborales) de los pueblos y, en particular, a "fomentar el progreso general de las naciones en vías de desarrollo", "socorrer en sus sufrimientos a los prófugos" o "ayudar a los inmigrantes y a sus familias" (GS 84).

En el ámbito de "la cooperación internacional en el campo económico" se subraya la imposibilidad de ayudar a los países pobres sin modificar las leyes vigentes del comercio internacional, indicando que, además, "con generosidad y sin ambición", "los países desarrollados deben prestar otras ayudas en forma de donativos, préstamos o inversiones" (GS 85b). Y entre las "normas oportunas" que propone GS 86 se reafirma "el deber gravísimo" de respetar las reglas de un comercio justo entre los países ricos y los pobres (cf. GS 86bc). En el mundo de hoy el antiguo proverbio "No le des peces, enséñale a pescar", que ha servido durante mucho tiempo para ilustrar las tareas de carácter promocional, es evidente que resulta insuficiente. Porque la gente ha aprendido a pescar y se sigue muriendo de hambre. Hoy ya no basta con saber pescar (hace falta también que te concedan la licencia de pesca, que las industrias no contaminen los ríos, que se pague lo justo al vender el pescado, que la familia del pescador tenga para comer cuando él ya no pueda pescar...).

También ante el fenómeno de la explosión demográfica de los pueblos del tercer mundo, la Constitución pastoral insiste en la urgencia de la cooperación de los países ricos. En concreto dice que contribuirían a

14 En 1965, mientras se debatía esta cuestión en el aula conciliar, un grupo internacional de 20 mujeres pertenecientes a la "comunidad del Arca", se trasladó a un convento romano y realizó un ayuno de 10 días -con permiso de Pablo VI- para pedir que Dios iluminara a los padres conciliares ante tan importante tema. 
mejorar mucho las condiciones de vida de los pobres poniendo a su disposición los avances de la técnica con el fin de "conseguir lo necesario para el sustento y la adecuada instrucción de los hombres" (GS 87a). Se reclama la ayuda técnica puesta al servicio de la alimentación y la educación de la infancia. Las políticas de control de la natalidad impuestas desde fuera a base de "gomas", además de inaceptables por injustas, sólo ponen "parches" y resuelven en falso el problema de la superpoblación. Por supuesto, hay que prevenir el SIDA y es necesaria la planificación familiar (GS 50b). Pero repartir condones alegremente no es más que una estrategia premeditada para tapar el problema de la pobreza y, lo que es más grave, para privar a los pobres de su única riqueza: la gente.

Como colofón, GS 90, el último número de la segunda parte de la Constitución pastoral, sienta las bases del organismo que encarna el compromiso permanente de la Iglesia con la causa de los pobres:

El Concilio, teniendo en cuenta la inmensidad de las calamidades que oprimen todavía ahora a la mayor parte de la humanidad y para fomentar en todas partes juntamente la justicia y el amor de Cristo hacia los pobres, juzga muy oportuna la creación de algún organismo de la Iglesia universal que tenga como misión estimular al conjunto de los católicos para promover el progreso de las regiones indigentes y la justicia social entre las naciones (GS 90c).

Este pasaje sugiere los dos principios fundacionales que inspirarán la creación de la Pontificia Comisión para la Justicia y la Paz"15: "fomentar en todas partes juntamente la justicia y el amor de Cristo hacia los pobres", o traducido al lenguaje secular, "promover el progreso de las regiones indigentes y la justicia social entre las naciones". Las múltiples iniciativas de este dicasterio velan por mantener vivo para la posteridad un legado inestimable de GS a la Iglesia.

\section{De la Iglesia en el mundo a la opción por los pobres}

A punto de terminar el Concilio, el 16 de noviembre de 1965, mientras en el aula conciliar se discutía todavía el esquema que se iba a convertir en la GS, un grupo de obispos, que se habían reunido periódica-

15 Motu proprio Catholicam Christi Ecclesiam (6 de enero de 1967): AAS 59 (1967) 27. Cf. PP 5; OA 52. Será elevada a Consejo Pontificio en 1976. 
mente en el Colegio Belga de Roma para estudiar el tema "L'Eglise et les pauvres", celebró una eucaristía en las catacumbas de santa Domitila. Con tal ocasión se hizo público un documento de 13 puntos conocido como "esquema XIV" o "pacto de las catacumbas". Redactado en portugués, fue presentado por mons. H. Cámara, arzobispo de Recife (Brasil). Es un precioso testimonio de la voluntad de la Iglesia del Vaticano II de convertirse a la causa de los pobres ${ }^{16}$.

Otros movimientos de renovación (como el bíblico, el litúrgico o el patrístico) habían preparado el Concilio y recibieron acogida oficial en sus documentos. Pero, en realidad, ha sido el movimiento de conversión de la Iglesia a los pobres el que, de una forma silenciosa, casi imperceptible en sus inicios y pese a ambigüedades y altibajos, ha marcado de un modo más decisivo los años del Postconcilio.

La mayoría de los autores coinciden en señalar dos períodos de recepción de GS. Durante la primera etapa (1966-1985), la Iglesia emprende un itinerario que asocia indisolublemente la tarea evangelizadora con el compromiso por los pobres ${ }^{17}$. Una trayectoria que avanza desde Populorum progressio (1967) y que desembocará, a través de Puebla (1979), en el Sínodo extraordinario sobre el Concilio (1985). A raíz de esa primera revisión oficial del Vaticano II se abría una nueva fase que ha tenido su último punto de referencia con ocasión del Jubileo. Repasamos los momentos más significativos de cada una, siempre en relación con nuestro tema.

\subsection{De Medellín a Roma}

Pablo VI fijaba la ruta a seguir en la encíclica Populorum progressio (PP): "nuestra caridad por los pobres del mundo -y son legión infinitadebe hacerse cada vez más atenta, más activa, más generosa" (PP 76). Este documento, que representa, junto con la Redemptor hominis (1979), el mejor comentario del magisterio a la $\mathrm{GS}^{18}$, va a acuñar la frase "los po-

16 Cf. J. L. Martín Descalzo, Un periodista en el Concilio IV, Madrid 196633, 490-493.

17 Cf. G.GIRARDI, "De la 'Iglesia en el mundo' a la 'Iglesia de los pobres'. El Vaticano II y la teología de la liberación" en C. FLORISTÁN - J.J. TAMAYO, eds., El Vaticano II, veinte años después, Madrid 1985, 429-463.

18 En 1995, con ocasión de los 30 años de la Constitución pastoral, Juan Pablo II, evocando su participación directa en el proceso de su elaboración, confesará que "proprio l'intima conoscenza della genesi della Gaudium et spes mi ha consentito di apprezzarne a fondo il valore profetico e di assumerne ampiamente i contenuti nel mio magistero dalla prima encíclica" y que en la Redemptor hominis recoge "la eredità della Costituzione conci- 
bres son cada vez más pobres, mientras los ricos se hacen cada vez más ricos" (PP 57) que hará fortuna en documentos pontificios sucesivos. Teniendo también a la Constitución pastoral como fuente directa de inspiración, pronto llegarán la carta apostólica Octogesima adveniens (1971) y los sínodos sobre la justicia en el mundo (1971) y sobre la evangelización (1975). Fruto de este último, es la exhortación Evangelii nuntiandi (1975). Este documento ofrece una visión integral de la evangelización donde se reconoce abiertamente en el anuncio evangélico un mensaje de liberación, que incluye como una tarea ineludible la promoción humana y la lucha por la justicia. Paralelamente, en este período tienen lugar las asambleas del episcopado latinoamericano de Medellín y Puebla.

La II Asamblea General del CELAM (Medellín 1968) significó la recepción del Vaticano II en América Latina y simultáneamente el alumbramiento de la teología de la liberación (TL). Dedicaba expresamente uno de sus documentos (14) al tema de la pobreza. En él, tras distinguir sus diversas acepciones (carencia de bienes, pobreza espiritual, compromiso voluntario) y proclamar el deseo de una Iglesia "evangelizadora de los pobres y solidaria con ellos", se habla ya de dar "preferencia efectiva a los sectores más pobres y necesitados y a los segregados por cualquier causa" (Pobreza n. 9), siguiendo el ejemplo de Cristo. Pero va a ser el documento final de la siguiente asamblea, celebrada en Puebla (1979), el que consagre oficialmente por primera vez la expresión "opción preferencial por los pobres" (OPP): "Afirmamos la necesidad de conversión de toda la Iglesia para una opción preferencial por los pobres, con miras a su liberación integral" (n. 1134). Es un tema recurrente en todo el documento, además de tener asignado un capítulo propio (el primero de la cuarta parte). Ahí se explica el significado de la expresión:

El compromiso evangélico de la Iglesia, como ha dicho el Papa, debe ser como el de Cristo: un compromiso con los más necesitados (cf. Lc 4,1821; Discurso inaugural III,3). La Iglesia debe mirar, por consiguiente, a Cristo cuando se pregunta cuál ha de ser su acción evangelizadora. El hijo de Dios demostró la grandeza de ese compromiso al hacerse hombre, pues se identificó con los hombres haciéndose uno de ellos, solidario con ellos, y asumiendo la situación en que se encuentran, en su nacimiento, en su vida y, sobre todo, en su pasión y muerte, donde llegó a la máxima expresión de la pobreza.

liare" (PONTIFICIO CONSIGLIO PER I LAICI, Gaudium et spes. Bilancio di un trentennio (Laici Oggi 39), Città del Vaticano 1996, 9-10). 
Por esta sola razón, los pobres merecen una atención preferencial, cualquiera que sea la situación moral o personal en que se encuentren. Hechos a imagen y semejanza de Dios para ser sus hijos, esta imagen está ensombrecida y aun escarnecida. Por eso Dios toma su defensa y los ama. Es así como los pobres son los primeros destinatarios de la misión y su evangelización es por excelencia señal y prueba de la misión de Jesús (nn.1141-1142).

La OPP nace en un contexto eclesial y con un contenido marcadamente cristológico, como el texto citado pone claramente de relieve. Se trata de una opción "exigida por la realidad escandalosa de los desequilibrios económicos" (n. 1154) sin otra finalidad que hacer a los pobres realmente partícipes del anuncio de Cristo Salvador (cf. n. 1153).

Inmediatamente la afortunada expresión va a encontrar eco en todas las latitudes de la Iglesia universal e incluso por parte de las otras iglesias cristianas, recibiendo el refrendo constante del Papa Juan Pablo II. A modo de ejemplo, podemos citar las enfáticas palabras del Pontífice en el mensaje de Navidad de 1984 al Colegio cardenalicio y a la Curia romana:

Aprovecho gustoso esta ocasión para reafirmar que el compromiso hacia los pobres constituye un motivo dominante de mi acción pastoral, la constante solicitud que acompaña mi servicio diario al pueblo de Dios. He hecho y hago mía tal "opción"; me identifico con ella. Y estimo que no podría ser de otra forma, ya que éste es el eterno mensaje del evangelio: así ha hecho Cristo, así han hecho los apóstoles de Cristo, así ha hecho la Iglesia a lo largo de su historia, dos veces milenaria ${ }^{19}$.

Al cumplirse el vigésimo aniversario de la clausura del Concilio se celebró en Roma el Sínodo extraordinario de los Obispos sobre el Vaticano II (1985). Representa la primera revisión oficial de la aplicación de sus enseñanzas a la vida de la Iglesia. En relación con la Constitución pastoral, el card. R. Etchegaray, presidente del Consejo Pontificio Iustitia et Pax, explicaba que la primera parte de GS había supuesto la reconciliación de la Iglesia no tanto con el mundo, sino más bien consigo misma.

19 "Un'opzione, inoltre, basata essenzialmente sulla parola di Dio e non su criteri apportati da scienze umane o ideologie contrapposte, che frequentemente riducono i poveri a categorie socio-politiche o economiche astratte. Un'opzione però decisa e irrevocabile" (Discorso ai cardinali e ai prelati della curia romana n. 9: Regno-doc. 3 (1985) 72). Cf. Discurso inaugural de la IV Asamblea del CELAM, n. 16 (Santo Domingo, 12 de Octubre de 1992). 
Según él, los dos últimos pontífices habían extraído de ella "no sólo el carácter ético de toda actividad social, sino el fundamento religioso de la ética social". Sobre el compromiso de la Iglesia del futuro con la causa de los pobres hay que reseñar la intervención del brasileño A. Lorscheider. Con la clarividencia de quien hablaba desde la propia experiencia pastoral volvía a auspiciar el tema roncalliano de la "Iglesia de los pobres", movida exclusivamente por la fidelidad a Cristo. Haciendo un balance de la recepción del texto conciliar el card. G. Danneels (Malinas) destacaba entre los aspectos positivos que la OPP había entrado a formar parte del pensamiento y de la praxis eclesial. En cambio, constataba entre los aspectos negativos el crecimiento de la pobreza y la miseria en los países pobres.

El Mensaje a los fieles, al invitar a la aplicación en profundidad de las cuatro constituciones del Vaticano II, proclama, parafraseando el párrafo inicial de la Constitución pastoral, que debe realizarse "en el servicio a los hombres y, sobre todo, a los pobres"20. Igualmente, tras expresar la voluntad "de llevar el Concilio a la práctica y a la vida de la Iglesia", el Documento final - "La Iglesia, bajo la Palabra de Dios, celebra el misterio de Cristo, para la salvación del mundo"- comienza empleando de nuevo el lenguaje de GS 1. En la última sección de su segunda parte, titulada "Misión de la Iglesia en el mundo", respecto a la actualidad de GS, señala que la situación de violencia y miseria que allí se constata sigue aumentando en el mundo, por lo que se requiere "una reflexión teológica nueva y más profunda que interprete tales signos a la luz del Evangelio". Posteriormente el documento sinodal aborda expresamente el tema de la "opción preferencial por los pobres y la promoción humana"21 y entre las sugerencias finales propone que se estudie cómo llevarlas a la práctica. En definitiva, el Sínodo acreditaba y respaldaba el anhelo profundo de la OPP que animaba a la TL.

\subsection{Del Segundo al Tercer milenio}

A mediados de los años 80', el apogeo de la TL, a pesar de sus detractores, hacía patente que se había producido un histórico cambio de perspectiva en la recepción de la Constitución pastoral. Había convertido a los

20 Nuntius ad Christifideles III en G. CAPRILE, Il Sinodo dei vescovi. Seconda assemblea generale straordinaria (24 novembre-8 dicembre 1985), Roma 1986, 551.

21 Cf. "Ecclesia sub Verbo Dei mysteria Christi celebrans pro salute mundi". Relatio finalis II, D, 6 (Ibid., 568-569). 
pobres en los primeros interlocutores de la misión de la Iglesia en el mundo 22 y a la opción preferencial por ellos en una exigencia de autoidentificación de la Iglesia, diferente de la auto-conciencia que había manifestado en el Vaticano II, cuando su mirada se había fijado mayormente en las sociedades modernas secularizadas.

Entre el Sínodo de 1985 y el Jubileo del 2000 se desarrolló un segundo período de recepción de GS, caracterizado, en palabras del card. J. Ratzinger, por "la búsqueda de un nuevo equilibrio, después de las exageraciones de una apertura indiscriminada al mundo moderno"23. A nivel mundial la Iglesia asistía al final de la "guerra fría" y a la hegemonía del sistema económico neoliberal a escala planetaria. Una etapa en la que precisamente el compromiso de la Iglesia con los pobres, impulsado poderosamente durante el largo pontificado de Juan Pablo II y asumido aparentemente por todos, sin embargo va a ser un factor determinante de la polarización de las posiciơnes dentro de la Iglesia, hacia el recelo de los nostálgicos de la restauración o hacia la decepción de los patrocinadores de un Vaticano III.

En aquel momento, la OPP se había generalizado en el lenguaje de la Iglesia postconciliar. Con más o menos matices, no hay documento del magisterio de la época que renuncie a su empleo. Incluso la primera Instrucción de la Sagrada Congregación para la Doctrina de la Fe, Libertatis nuntius (1984), que cuestionaba ciertas "desviaciones" de la TL, tuvo buen cuidado en comenzar aclarando que "esta llamada de atención de ninguna manera debe interpretarse como una desautorización de todos aquellos que quieren responder generosamente y con auténtico espíritu evangélico a la opción preferencial por los pobres".

En numerosas ocasiones el magisterio de Juan Pablo II ha reiterado la predilección de la Iglesia por los pobres ${ }^{24}$. Según la Carta encíclica Sollicitudo rei socialis (SRS), conmemorativa del vigésimo aniversario (1987) de Populorum progressio, las palabras iniciales de GS expresan el "motivo fundamental" inspirador de todo el documento. Se parte de la

22 Se hablaba entonces de la irrupción de los pobres como "nuevo sujeto histórico". Cf. G.Girardi, "De la 'Iglesia en el mundo' a la 'Iglesia de los pobres'. El Vaticano II y la teología de la liberación", 445-446; L. SARTORI, "Sviluppi delle coordinate teologiche della Gaudium et spes" en La costituzione conciliare Gaudium et Spes vent'anni dopo, Padova 1988, 42-43.

23 J. RATZINGER-V. MESSORI, Informe sobre la fe, Madrid 1985, 72.

24 Indica esa precedencia empleando, además de "prasertim", otras expresiones como "imprimis" "anteponens", "optio", "amor potior", "cura praecipua", etc. 
constatación del estado de "miseria" en el que viven millones de seres humanos. Frente a esa situación, se recuerda que la Constitución pastoral quiere decir una palabra de "gozo y esperanza" (SRS 6). En esta encíclica el Papa considera que la perspectiva de la liberación se ha convertido en un "nuevo modo" de afrontar aquellas situaciones y en un principio de acción "noble y válido" (SRS 46). SRS 42 describe el alcance de "la opción o amor preferencial por los pobres" a cuatro niveles (personal, social, nacional e internacional):

Esta es una opción o una forma especial de primacía en el ejercicio de la caridad cristiana, de la cual da testimonio toda la tradición de la Iglesia. Se refiere a la vida de cada cristiano, en cuanto imitador de la vida de Cristo, pero se aplica igualmente a nuestras responsabilidades sociales y, consiguientemente, a nuestro modo de vivir y a las decisiones que se deben tomar coherentemente sobre la propiedad y el uso de los bienes.

Pero hoy, vista la dimensión mundial que ha adquirido la cuestión social, este amor preferencial... no puede dejar de abarcar a las inmensas muchedumbres de hambrientos, mendigos, sin techo, sin cuidados médicos y, sobre todo, sin esperanza de un futuro mejor: no se puede olvidar la existencia de esta realidad. Ignorarlo significaría parecernos al "rico epulón", que fingía no conocer al mendigo Lázaro, postrado a su puerta (cf. Lc 16,19-31).

Nuestra vida cotidiana, así como nuestras decisiones en el campo político y económico, deben estar marcadas por estas realidades. Igualmente los responsables de las naciones y los mismos organismos internacionales... no han de olvidar dar la precedencia al fenómeno de la creciente pobreza. Por desgracia, los pobres, lejos de disminuir, se multiplican no sólo en los países menos desarrollados, sino también en los más desarrollados, lo cual resulta no menos escandaloso (SRS 42bcd).

En la carta apostólica Tertio millennio adveniente (TMA) de 1994, con vistas a la celebración del año jubilar, el Papa identificaba en el diálogo "abierto, respetuoso y cordial" con el mundo y en el atento discernimiento en el testimonio de la verdad la tarea legada por la Constitución pastoral (cf. TMA 36). Y para el último año de la fase preparatoria del Jubileo, "recordando que Jesús vino a evangelizar a los pobres (Mt 11,5; Lc 7,22)", indicaba el imperativo de "subrayar más decididamente la opción preferencial de la Iglesia por los pobres y los marginados" mediante iniciativas concretas (p.e. condonación de la deuda).

Diferentes estudios aparecidos en la última década insinúan que con el Jubileo del nuevo milenio se habría inaugurado un nuevo período de 
recepción de la GS ${ }^{25}$. Sea como fuere, lo cierto es que nuestro tema mereció una atención muy particular por parte de la carta apostólica con motivo de tal evento (2001). En efecto, el programa diseñado en Novo Millennio Ineunte (NMI) reconoce que el Concilio Vaticano II ha sido una gracia para la Iglesia del s. XX y nos ofrece "una brújula segura para orientarnos en el camino del siglo que comienza" (NMI 57). Este documento repasa "el panorama de la pobreza" en el mundo, las pobrezas de siempre y las nuevas (cf. NMI 50), y augura una apuesta más activa y concreta de la Iglesia por los más pobres en el milenio que se estrenaba:

Si verdaderamente hemos partido de la contemplación de Cristo, tenemos que saberlo descubrir sobre todo en el rostro de aquéllos con los que El mismo ha querido identificarse... (cita Mt 25,35-36). Esta página no es una simple invitación a la caridad: es una página de cristología, que ilumina el misterio de Cristo. Sobre esta página, la Iglesia comprueba su fidelidad como esposa de Cristo, no menos que sobre el ámbito de la ortodoxia $^{26}$.

25 Cf. V. Botella Cubells, El Vaticano II ante el reto del tercer milenio. Hermenéutica y Teología, Salamanca-Madrid 1999, 21-52; L. BoEVE, "Gaudium et spes and the crisis of Modernity: the end of the dialogue with the World?" en M. LAMBERIGTS - L. KENIS, eds., Vatican II and its Legacy, Louvain 2002, 83-94; N. METre, "Gaudium et Spes - Die Pastoralkonstitution und das Pastoralkonzil": Münchener Theologische Zeitschrift 54 (2003) 114-126; D. MolinA, "Iglesia-mundo: una relación cambiante": Sal Terrae 92 (2004) 163-174; S. MADRIGAL, "Las relaciones Iglesia-mundo según el Concilio Vaticano II" en G. URf́BARRI, ed., Teología y Nueva Evangelización, Bilbao 2005, 13-95; E. BorGMAN, "Gaudium et spes: el futuro olvidado de un documento revolucionario": Concilium 312 (2005) 499-508; G. ROUTHIER, Vatican II: hermeneutique et reception, Québec 2006; A. MELLONI-G. RUGGIERI, Chi ha paura del Vaticano II?, Roma 2009; C. THEOBALD, La réception du concile Vatican II. I. Accéder à la source, Paris 2009; S. MADRIGAL, Unas lecciones sobre el Vaticano II y su legado, 195-206; M. VIDAL, "Recepción y hermenéutica del Concilio Vaticano II": Moralia 35 (2012) 375-406; el monográfico sobre los cincuenta años del Vaticano II de Concilium 346 (2012), especialmente los arts. de P. Hünermann (319-333) y J. Sobrino (395-405).

26 En el discurso de apertura de la IV Asamblea del Consejo Ecuménico de las Iglesias (Uppsala 1968), su presidente Visser’t Hooft presentó la cuestión de la opción por los pobres de la siguiente manera: "un cristianismo que haya perdido su dimensión vertical se habrá perdido a sí mismo. Pero un cristianismo que utilice las preocupaciones verticales como medio para rehuir responsabilidades ante los hombres no será ni más ni menos que una negación de la encarnación. Es hora de comprender que todo miembro de la Iglesia que rehuya en la práctica tener una responsabilidad ante los pobres es tan culpable de herejía como el que rechaza una de las verdades de la fe" (citado por A. GARCía Lucio, "La caridad política: motor del compromiso social": Corintios XIII (1998) 52). Por su parte, I. Ellacuría decía que "la OPP es una de las notas de la verdadera Iglesia, al nivel de aquellas que antiguamente definíamos como una, santa, católica y apostólica" (I. ElLACURía, "Las Iglesias latinoamericanas interpelan a la Iglesia de España”: Sal Terrae 70 (1982) 221). 
No debe olvidarse, ciertamente, que nadie puede ser excluido de nuestro amor, desde el momento que "con la encarnación el Hijo de Dios se ha unido en cierto modo a cada hombre" [GS 22b]. Ateniéndonos a las indiscutibles palabras del Evangelio, en la persona de los pobres hay una presencia especial suya, que impone a la Iglesia una opción preferencial por ellos (NMI 49).

Es sumamente importante destacar que en este párrafo Juan Pablo II volvía a insistir en el fundamento cristológico de la opción por los pobres enlazando el pasaje de Mt 25 con la cita de GS 22, probablemente el texto más rico producido por el Vaticano II desde el punto de vista de la cristología y uno de los más comentados en el Postconcilio. Seguidamente, Juan Pablo II proclama que es la hora de "una nueva imaginación de la caridad" para que los pobres se sientan en la Iglesia como "en su casa", propugnando un estilo más eficaz y creíble de evangelización en el que necesariamente las obras deben seguir a las palabras (NMI 50).

Su sucesor, Benedicto XVI (2005-2012), ha dado pasos importantes para dotar de nuevos impulsos a la tarea de una nueva evangelización (NE), ya emprendida por los papas anteriores. Desde su primera encíclica (2005), Dios es amor (DCE), su magisterio ha puesto de relieve que uno de los lugares privilegiados de la NE es el de la caridad y, "sobre todo", la caridad hacia los pobres 27 . En el discurso inaugural de la V Asamblea del Episcopado Latinoamericano (2007), Benedicto XVI subrayaba de nuevo el fundamento cristológico de la OPP: "La opción preferencial por los pobres está implícita en la fe cristológica en aquel Dios que se ha hecho pobre por nosotros, para enriquecernos con su pobreza (cf. 2 Cor 8,9)". Y dirigiéndose en particular a los religiosos y religiosas, les daba las gracias en nombre de la Iglesia de América Latina por su trabajo en favor de sus hermanos "sobre todo de los más pobres y marginados"28.

En cualquier caso, no nos equivocamos al afirmar que va a ser el primer pontífice latinoamericano, el Papa Francisco I, el que va a dar el impulso más decisivo a la misión de la Iglesia en el mundo de los pobres.

27 Cf. R. Fisichella, La nueva evangelización, Santander 2012, 68-73.

28 BeNEDICTO XVI, Discurso inaugural de la V Asamblea de la Conferencia del Episcopado Latinoamericano (Aparecida, 13 de mayo de 2007) en www.vatican.va. El carácter cristológico de la "opción preferencial por los pobres y excluidos" va ha quedar plasmado inequívocamente en la redacción final del Documento de Aparecida (nn. 257; 391-396; 550). 


\section{Conclusiones}

Siendo fieles a la "hermenéutica de la reforma" o "de la renovación en la continuidad" que propugnaba Benedicto XVI en su mensaje de Navidad a la Curia al evocar los 40 años del Vaticano $\mathrm{II}^{29}$, de lo expuesto se derivan importantes consecuencias para la misión pastoral de la Iglesia, no sólo desde el punto de vista de la moral cristiana, sino también de la teología fundamental. Enumeramos algunas de ellas a modo de conclusión.

1. En el campo de la moral social, GS representa un punto de inflexión, sancionado con la autoridad de un Concilio, de la ampliación de horizontes respecto a las mediaciones de la caridad. Junto a la labor asistencial y las acciones de promoción humana (cf. GS 42b; 69a), que han sido una constante en la obra social de la Iglesia a lo largo de la historia, hoy las situaciones de pobreza y marginación reclaman, además, una especial atención a las mediaciones políticas (cf. GS 76e). La dimensión política de la caridad requiere abordar en profundidad las causas y no sólo los efectos de las injusticias y desigualdades. Ya Pío XI habló de la "caridad política". Juan Pablo II lo ha denominado "una nueva imaginación de la caridad". M. L. King, comentando la parábola del buen Samaritano, decía que ante el caminante herido también es amor denunciar el estado del camino de Jerusalén a Jericó para que no se repitan los asaltos ${ }^{30}$.

2. La atención prioritaria de la Iglesia por los pobres, con las implicaciones para la ética individual y social que de ella se desprenden, se basa, ante todo, en presupuestos cristológicos y eclesiológicos que traspasan los postulados de la razón o del derecho natural. Ello no quiere decir que GS represente una "ruptura" respecto a la llamada "doctrina social de la Iglesia" (su segunda parte se sitúa en clara continuidad con ella). Pero sí subraya que tiene un rango y un enfoque diverso; en absoluto se trata de otro documento social más 31 .

29 ID., Discurso a la Curia Romana 22 de diciembre de 2005 en Insegnamenti di Benedetto XVI I (2009), 1023-1024.

30 M. L. KING, La fuerza de amar, Barcelona $1973^{11}, 28$, citado por L. GoNZÁLEZCARVAJAL, Con los pobres contra la pobreza, Madrid 1992², 120.

31 Evidentemente no lo entendía así cierto teólogo "neoliberal" que en el Encuentro Internacional conmemorativo de los 30 años de GS, celebrado en Loreto, comenzó su ponencia diciendo literalmente que "hoy la Gaudium et Spes debe ser leída a la luz de la Centesimus Annus" (M. NovaK, "Attenti al trionfalismo!" en Gaudium et Spes. Bilancio di un trentennio, 82). 
3. Si como se ha dicho, según la antropología de GS, "Cristo entra en la definición del hombre" (cf. GS 22ab), Él entra especialmente en la definición del pobre por cuya voz, además, sigue hablando personalmente (cf. GS 88a). La OPP es una opción cristológica. No hace falta decir que la opción primordial de la Iglesia es por Cristo. Lo que hay que recordar es que ésa incluye indisolublemente la opción por los pobres. Por consiguiente, dicha opción (que nunca es excluyente), si se puede decir así, tampoco es opcional. No lo fue, por ejemplo, para la beata Madre Teresa cuando en los barrios de Calcuta veía al propio Cristo en los más pobres entre los pobres, como es sobradamente conocido (cf. DCE 18).

4. Conviene tener siempre presente que GS no es la Constitución conciliar sobre "el mundo actual", ni siquiera sobre "la Iglesia y el mundo", sino sobre "la Iglesia en el mundo". La Iglesia no está más allá o por encima del mundo, sino que se encuentra en medio del mundo como sacramento universal de salvación (cf. LG 48; GS 45a) allí donde los discípulos de Cristo hacen propias las esperanzas y angustias del género humano, sobre todo de los pobres, en cada momento de la historia. Esta Iglesia que se reconoce a sí misma en su misión entre los pobres es seguramente el aspecto menos explorado que se deriva de la eclesiología del Vaticano II y cuyas consecuencias están todavía por desentrañar. Algo vislumbró Mons. Romero hasta dar la vida 32 .

Como homenaje al Papa Francisco I, "el Papa de los pobres", al comienzo hemos recordando a Francisco de Asís, paradigma de la santidad entre los pobres. Hemos citado también a tres profetas contemporáneos (M. L. King, Teresa de Calcuta y O. Romero). Terminamos evocando el testimonio de otro santo. En este caso del primer milenio, el mártir Lorenzo. Su figura fue repropuesta como modelo, entre otras, por Benedicto XVI (cf. DCE 23). Otro Pontífice, en camino de los altares, el Papa Juan Pablo I, a pesar de su brevísimo pontificado, tuvo también ocasión de rendirle homenaje. Lo hizo con estas sencillas -y proféticas- palabras:

Roma será una verdadera comunidad cristiana si se honra en ella a Dios no sólo con la afluencia de los fieles a las iglesias, ni únicamente con la vida privada vivida con moderación, sino también con el amor a los

32 Es muy elocuente su discurso de investidura como doctor honoris causa en la Universidad de Lovaina, cf. O. A. ROMERo, "La dimensión política de la fe desde la opción por los pobres" (2 de febrero de 1980), citado por J.I. GonZÁlez FAUs, Vicarios de Cristo, Los pobres en la teología y la espiritualidad cristianas, Madrid 1991, 333-337. 
pobres. Estos -decía el diácono romano Lorenzo- son los verdaderos tesoros de la Iglesia 33 .

“AAh, cómo quisiera una Iglesia pobre y para los pobres!”, exclamaba espontáneamente el Papa Francisco, apenas recién elegido, recuperando el viejo sueño roncalliano ${ }^{34}$, en su primer encuentro con los responsables de los medios de comunicación. Y es que, cuando mira al mundo, la Iglesia tiene que mirar sobre todo - praesertim - a los pobres. Con la perspectiva del medio siglo transcurrido, podemos afirmar que una Iglesia decidida a ser pobre y para los pobres es una de las principales adquisiciones del Vaticano II. En su Constitución pastoral la Iglesia se ha manifestado como defensora de los pobres, justificando con su autoridad las reivindicaciones de ellos ${ }^{35}$. La solidaridad con los pobres ha sido desde la antigüedad uno de los empeños principales de la Iglesia. Especialmente hoy es el testimonio más claro de una fe viva y, para quienes no pertenecen a la Iglesia, se ha convertido en un criterio inequívoco de su credibilidad.

33 JUAN PABLo I, "Autoridad y libertad en la Iglesia": Ecclesia 1904 (1978) 9.

34 "La Iglesia se presenta como es, y quiere ser, como la Iglesia de todos y particularmente la Iglesia de los pobres" ("Ecclesia Christi lumen gentium" en Discorsi, messaggi, colloqui del Santo Padre Giovanni XXIII, IV, 524). La aplaudida intervención del card. G. Lercaro en el aula conciliar la víspera de la clausura del primer período de sesiones (cf. Acta Synodalia I, IV, 327-330) recogía aquel mismo deseo.

35 Cf. J. B. METZ, "Die Christen in der Welt von Heute" en Reformation aus Rom. Die katholische Kirche nach dem Konzil, Tübingen 1967, 79-81; J. LLIGADAS, "Las siete principales adquisiciones del Concilio" en Concilio Vaticano II, una llamada de futuro, Barcelona 2012, 48-49. 